\title{
Hall-effect evolution across a heavy-fermion quantum critical point
}

\author{
S. Paschen*, T. Lühmann*, S. Wirth*, P. Gegenwart*, O. Trovarelli*, C. Geibel*, \\ F. Steglich*, P. Coleman ${ }^{\dagger} \&$ Q. $\mathrm{Si}^{\ddagger}$ \\ * Max Planck Institute for Chemical Physics of Solids, Nöthnitzer Str. 40, D - \\ 01187 Dresden, Germany \\ ${ }^{\dagger}$ Center for Materials Theory, Department of Physics and Astronomy, Rutgers University, \\ Piscataway, New Jersey 08855, USA \\ ¥Department of Physics and Astronomy, Rice University, Houston, Texas 77005-1892, USA
}

\begin{abstract}
A quantum critical point (QCP) develops in a material at absolute zero when a new form of order smoothly emerges in its ground state. QCPs are of great current interest because of their singular ability to influence the finite temperature properties of materials. Recently, heavy-fermion metals have played a key role in the study of antiferromagnetic QCPs. To accommodate the heavy electrons, the Fermi surface of the heavy-fermion paramagnet is larger than that of an antiferromagnet $\frac{1,2,3}{}$. An important unsolved question concerns whether the Fermi surface transformation at the QCP develops gradually, as expected if the magnetism is of spin density wave (SDW) type ${ }^{4.5}$, or suddenly as expected if the heavy electrons are abruptly localized by magnetism ${ }^{6.7 .8}$. Here we report measurements of the low-temperature Hall coefficient $\left(R_{H}\right)$ - a measure of the Fermi surface volume - in the heavy-fermion metal $\mathrm{YbRh}_{2} \mathrm{Si}_{2}$ upon field-tuning it from an antiferromagnetic to a paramagnetic state. $R_{H}$ undergoes an increasingly rapid change near the QCP as the temperature is lowered, extrapolating to a sudden jump in the zero temperature limit. We interpret these results in terms of a collapse of the large Fermi surface and of the heavy-fermion state itself precisely at the QCP.
\end{abstract}


The compound $\mathrm{YbRh}_{2} \mathrm{Si}_{2}$ investigated here appears to be one of the best suited heavyfermion metals known to date to study the evolution of the Hall effect across a QCP. Magnetic susceptibility and specific heat indicate that it orders antiferromagnetically via a second-order phase transition at very low temperatures $\left(T_{N}=70 \mathrm{mK}\right)^{9}$. The antiferromagnetic nature of the transition is supported by NMR data $\frac{10}{}$. Neutron scattering experiments to directly detect the magnetic order are, presumably due to the smallness of the ordered moment $\underline{\underline{11}}$, not available to date. The Néel temperature $T_{N}$ is continuously suppressed down to the lowest experimentally accessed temperatures by application of a small magnetic field $\left(B_{1 c} \approx 0.7 \mathrm{~T}\right.$ for a field along the magnetically hard $c$-axis, $B_{2 c} \approx 60 \mathrm{mT}$ for a field within the easy tetragonal plane $)^{12}$. In addition, isothermal magnetostriction measurements indicate that the transition remains of second order down to at least $15 \mathrm{mK}$ (ref. 13). Although a change from second to first order at even lower temperatures can, of course, not be strictly ruled out the non-Fermi liquid behaviour observed for three decades of temperature $(10 \mathrm{mK}<T<10 \mathrm{~K})^{\underline{12}}$ is best described within a quantum critical picture. The use of tiny fields permits one to reversibly access the QCP without the introduction of additional disorder and without altering the character of the underlying zero-field transition $\underline{14}$. Moreover, unlike for several other heavy-fermion compounds 15 (and the high- $T_{c}$ superconductors), the QCP is not hidden by superconductivity. This is in spite of the high quality of the $\mathrm{YbRh}_{2} \mathrm{Si}_{2}$ single crystals investigated here (residual resistivities of $\approx 1 \mu \Omega \mathrm{cm}$, ref. 12 ). The scaling analysis of the thermodynamic and dynamical properties (specific heat, magnetic susceptibility, electrical resistivity) suggests $\frac{12}{2}$ that the field-induced QCP in $\mathrm{YbRh}_{2} \mathrm{Si}_{2}$ is of local ${ }^{6.7 .8}$ rather than of itinerant ${ }^{4.5}$ type, similar to the doping-induced QCP in $\mathrm{CeCu}_{6-x} \mathrm{Au}_{x}$ (ref. 6). Hall-effect measurements may be used to access a static electronic property, namely the Fermi surface volume, for which clear-cut theoretical predictions exist for different types of QCP $\underline{7.16}$. The study presented here is the first systematic Hall-effect measurement at a heavy-fermion QCP.

The Hall effect, usually a rather complex quantity, appears to be surprisingly simple here, both in vanishingly small and in finite magnetic fields. Outside the quantum critical region the Hall resistivity is linear in field resembling the behaviour of simple metals. Furthermore, our analysis of the temperature-dependent Hall coefficient in terms of the anomalous Hall effect (Fig. पa, Methods, and refs. 17 and 18) reveals that the low-temperature (below about $1 \mathrm{~K})$ Hall coefficient is dominated by its normal contribution. These features imply that 
the low-temperature Hall coefficient can be used, to a good approximation, as a measure of the Fermi surface volume. In the absence of photoemission and de Haas-van Alphen studies (the latter presumably never being available because of the very low critical magnetic field) as well as of electronic bandstructure calculations this is, so far, the only information on the Fermi surface volume of $\mathrm{YbRh}_{2} \mathrm{Si}_{2}$. At zero magnetic field, the data measured at the lowest temperatures tend to saturate at the value of the normal Hall coefficient extracted from the data between $7 \mathrm{~K}$ and room temperature (Fig. 19). This indicates that, at $B=0$, the Fermi surface volume is the same at the lowest temperatures as it is at high temperatures. Thus, even though there is evidence for the onset of Kondo screening at approximately $20 \mathrm{~K}$ (refs. 9 and 12) and for surprisingly large effective quasiparticle masses in the antiferromagnetically ordered state close to the $\mathrm{QCP}^{12}$, the local moments do, at the lowest temperatures and at $B=0$, not appear to be incorporated into the Fermi surface. In the static sense ${ }^{1.2 .3}$, $\mathrm{YbRh}_{2} \mathrm{Si}_{2}$ may, in its unconventional antiferromagnetic state at $B<B_{c}$, therefore not be classified as a "heavy-fermion" metal.

In the intermediate temperature range, between approximately $70 \mathrm{mK}$ and $7 \mathrm{~K}$, there is an additional contribution $\Delta R_{H}$ which is not due to the anomalous Hall effect (Fig. 19). In the main part of Fig.1 $1 \mathrm{~b}$ we show that, between 0.7 and $5 \mathrm{~K}$, the cotangent of the Hall angle is linear in $T^{2}$ (while $\Delta \rho \propto T$ ), indicating that the longitudinal and transverse scattering rates are different ${ }^{19}$. This type of behaviour is well known in high- $T_{c}$ cuprates where it has been taken as evidence for spin-charge separation ${ }^{19}$. Note, however, that for $\mathrm{YbRh}_{2} \mathrm{Si}_{2}$ the temperature range where this relation holds (inset of Fig. (1)), is narrower than the one where the non-Fermi liquid (NFL) behaviour $\Delta \rho \propto T$ is observed (from $100 \mathrm{mK}$ to almost $10 \mathrm{~K}$, ref. 12), even if $\mathrm{YbRh}_{2} \mathrm{Si}_{2}$ is field-tuned to quantum criticality (green squares in inset). The same may hold true for $\mathrm{CeCoIn}_{5}$ (ref. 20).

In our field-dependent Hall-effect measurements on $\mathrm{YbRh}_{2} \mathrm{Si}_{2}$ the magnetic field plays dual roles, as both a "tuning" and a "probe" field. On the one hand, the coupling between the field and the $\mathrm{Yb}^{3+}$ moments tends to align the latter: it is this Zeeman-like coupling which tunes the ground state of the material, ultimately suppressing the antiferromagnetism and creating the QCP. On the other hand, the magnetic field also generates a weak Lorentz force on the underlying electrons which produces the Hall response. The weak orbital coupling responsible for the Lorentz force does not appreciably change the ground state so that, to a good approximation, we can discuss the two couplings independently. The single 
crystals of $\mathrm{YbRh}_{2} \mathrm{Si}_{2}$ are thin platelets oriented along the $a b$-plane and practical Hall-effect measurements require a current and Hall voltage lying in this plane. This allows for two distinct types of experiment, "transverse tuning" where the tuning field $B_{1}$ is parallel to the $c$-axis, perpendicular to the current, and "longitudinal tuning" where the tuning field $B_{2}$ lies parallel to the current in the basal plane (cf. schematics in Figs.2a and b). The longitudinal field $B_{2}$ produces essentially no Hall response (see Supplementary Methods 1) and serves only to tune the state: a separate, crossed probe field $\delta B_{1}$ along the $c$-axis is required to measure the Hall response. In this longitudinal (crossed-field) experiment, the Hall resistivity $\rho_{H}$ is a direct measure of the field-tuned (linear-response) Hall coefficient $R_{H}\left(B_{2}\right)$

$$
R_{H}\left(B_{2}\right) \equiv \lim _{B_{1} \rightarrow 0} \rho_{H}\left(B_{2}, B_{1}\right) / B_{1} .
$$

In the transverse (single-field) case, on the other hand, the magnetic field simultaneously tunes the state and probes the Hall response and the differential Hall coefficient $\tilde{R}_{H}\left(B_{1}\right)$ is

$$
\tilde{R}_{H}\left(B_{1}\right) \equiv \frac{d \rho_{H}\left(B_{1}\right)}{d B_{1}}=\left[\frac{\partial \rho_{H}\left(B_{1}\right)}{\partial B_{1}}\right]_{\text {orb }}+\left[\frac{\partial \rho_{H}\left(B_{1}\right)}{\partial B_{1}}\right]_{\text {zeeman }}=R_{H}\left(B_{1}\right)+\left[\frac{\partial \rho_{H}\left(B_{1}\right)}{\partial B_{1}}\right]_{\text {zeeman }} .
$$

The orbital ("probing") contribution is, according to the Kubo formalism, just the generalized definition of a Hall coefficient (see Supplementary Methods 2). The Zeeman ("tuning") term is not related to a readily measurable linear-response quantity.

We first discuss the results of the single-field experiment. Figure2 a displays several representative isotherms of the Hall resistivity $\rho_{H}$, corrected for its anomalous contribution $\rho_{H, a}(B)$ (see Methods), vs $B_{1} \cdot \rho_{H}-\rho_{H, a}$ shows a linear low- $B_{1}$ behaviour with larger and a linear high- $B_{1}$ behaviour with smaller slope. The crossover between the two regimes broadens and shifts to higher $B_{1}$ with increasing temperature. For a quantitative analysis of the data we choose $\tilde{R}_{H}(B)=R_{H}^{\infty}-\left(R_{H}^{\infty}-R_{H}^{0}\right) \gamma(B)$ as a fitting function, where $R_{H}^{0}$ is the zero-field Hall coefficient and $R_{H}^{\infty}$ is the asymptotic differential Hall coefficient at large fields. $\gamma(B)$ is a crossover function that changes from unity at low fields to zero at large fields, which we parameterize as $\gamma(B)=1 /\left[1+\left(B / B_{0}\right)^{p}\right]$. Here, $B_{0}$ is the crossover field and $p$ determines the sharpness of the transition, which has a width $\Gamma \sim B_{0} / p$ when $p$ is large. For $p \rightarrow \infty$, $\int \tilde{R}_{H}(B) d B$ has a sharp kink at $B=B_{0}$, corresponding to a step in $\tilde{R}_{H}(B)$ itself. The fits to the data are shown as solid lines in Fig.21. For one temperature the derivative of the fit, corresponding to $\tilde{R}_{H}\left(B_{1}\right)$, is shown as well. The crossover fields $B_{0}$ obtained from these fits are included as red dots in the temperature-field $(T-B)$ phase diagram of $\mathrm{YbRh}_{2} \mathrm{Si}_{2}$ 
(Fig.[3 $\mathrm{k}$ ). A linear fit to these points (dashed red line in Fig.[3a denoted $T_{\text {Hall }}$ ) extrapolates at zero temperature to the critical field $B_{1 c} \approx 0.7 \mathrm{~T}$ for the disappearance of antiferromagnetic order and the QCP. Thus, the crossover is directly related to the QCP. The sharpness of the crossover is best quantified by the full width at half maximum (FWHM) of $d \tilde{R}_{H} / d B_{1}$, which represents the change of slope of $\rho_{H}\left(B_{1}\right)$. The temperature dependence of the FWHM values is well described by a pure power law, FWHM $\propto T^{a}, a=0.5 \pm 0.1$ (inset of Fig. Ba), suggesting that, at zero temperature, the crossover is infinitely sharp $(\mathrm{FWHM}=0)$.

To understand the origin of this sharp feature, we now turn to the crossed-field measurement of the linear-response Hall coefficient, Eq. (11). The inset of Fig.20 displays $\rho_{H}\left(B_{1}\right)$ curves taken at $65 \mathrm{mK}$ for different values of the longitudinal tuning field $B_{2}$. With increasing $B_{2}$ the linear-response Hall coefficient $R_{H}$ decreases. For a quantitative analysis we fit, as above, $\int \tilde{R}_{H}(B) d B$ to the $\rho_{H}\left(B_{1}\right)$ data (solid lines in the inset of Fig.2 $\mathrm{b}$ ). As opposed to the single-field experiment, $R_{H}^{0}=R_{H}$ is now the only parameter to consider. $R_{H}$, normalized to its value at the crossover field $B_{0}$, is plotted in the main panel of Fig. 20 as a function of the normalized tuning field $B_{2} / B_{0}$. Data obtained in the same way at 45,75 , and $93 \mathrm{mK}$ are included as well. $R_{H}$ decreases as a function of $B_{2}$ by a factor of $\approx 1.5$. In a simple one band model this corresponds to an increase in the charge carrier concentration from $\approx 2$ to $\approx 3$ holes per $\mathrm{YbRh}_{2} \mathrm{Si}_{2}$ formula unit. The crossover sharpens up as the temperature is lowered. For a quantitative analysis we may now fit the crossover form $R_{H}(B)=R_{H}^{\infty}-\left(R_{H}^{\infty}-R_{H}^{0}\right) \gamma(B)$ to the $R_{H}\left(B_{2}\right)$ data (solid curves in main panel of Fig. (2) ). The $R_{H}^{\infty}$ values obtained for these four temperatures are included as green triangles in the main part of Fig. [a, showing that the Hall coefficient in the field-induced Landau Fermi liquid (LFL) state (cf. Fig. 3a ) at very low temperatures is substantially smaller than in the $B=0$ antiferromagnetically ordered state. The $11 B_{0}$ and FWHM values obtained from the above fits are included as green dots in Fig. [3a and its inset. The factor of 11 accounts for the fact that the tuning field $B_{2}$ is applied in the easy tetragonal plane of $\mathrm{YbRh}_{2} \mathrm{Si}_{2}$ where, due to the magnetic anisotropy, the action of a magnetic field is known to be $\approx 11$ times as strong as along the magnetically hard $c$-axis $\frac{12}{2}$. For both quantities the green and red data points agree within the error bars. Thus, the linear Hall response $R_{H}\left(B_{2}\right)$ of the crossed-field measurement and the differential Hall response $\tilde{R}_{H}\left(B_{1}\right)$ of the single-field measurement can be described by the same functional form and the respective crossover positions and crossover widths agree quantitatively. This experimental finding suggests that the second term on the right hand 
side of Eq. (2) plays a minor role, at least in the experimentally accessed part of the $T-B$ phase diagram. Therefore, here the single-field experiment appears to probe essentially the same (linear-response) Hall coefficient as the crossed-field experiment. However, there is a quantitative difference in the jump heights of $\tilde{R}_{H}\left(B_{1}\right)$ and $R_{H}\left(B_{2}\right)$ which probably reflects the anisotropies in the evolution of the electronic bandstructure under transverse and longitudinal field-tuning 21 , amplified by the likely presence of a multisheeted, anisotropic Fermi surface.

The phase diagram in the magnetic field-temperature parameter space can now be illustrated by a 3D representation of $d \gamma(B) / d B$ (Fig. B $3 \mathrm{~b}$ ). $\gamma(B)$ is calculated at arbitrary temperatures from the linear $B_{0}$ vs $T$ fit (dashed red line in Fig. [3 $\mathrm{k}$ ) and a power law fit (not shown) to the $p(T)$ data obtained from the fits to $\rho_{H}\left(B_{1}\right)$ (Fig. 22 ) and to $R_{H}\left(B_{2}\right)$ (main panel of Fig.2b). With decreasing temperature, the $d \gamma(B) / d B$ curves sharpen up and their crossover position $B_{0}$, designated by drop lines, shifts to lower fields such that, at zero temperature, a $\delta$-function (dashed line in $T=0$ plane in Fig. (3b) is situated at the QCP.

Thus, the extrapolation of our finite temperature data to zero temperature indicates the presence of a finite discontinuity ("jump") in the Hall coefficient at the QCP, even though the change in the magnetic order parameter is infinitesimall $1 \underline{11}$. By contrast, in an itinerant SDW scenario, the Fermi surface is expected ${ }^{7}$ to fold over at the QCP; the Hall coefficient is then continuous across the QCP, evolving gradually with the size of the antiferromagnetic order parameter, as is indeed observed experimentally $\underline{22}$. (For a more quantitative comparison, see caption of Fig.2b.) Our results hint at a sudden reconstruction of the Fermi surface at the QCP, corresponding to the sudden loss of "mobile" $4 f$ electrons ${ }^{7.8 .16}$. Loosely speaking, the volume of the Fermi surface has changed discontinuously. Here of course, the concept of Fermi surface volume needs to be treated with some care, for antiferromagnetism breaks translational symmetry. $\mathrm{YbRh}_{2} \mathrm{Si}_{2}$ may well be an easy-plane incommensurate antiferromagnet, and for this class of antiferromagnet, to linear order in the magnetic order parameter, the Fermi surface volume is well-defined in the paramagnetic unit cell. From our data we infer that the antiferromagnetic ground state has a "small" Fermi surface which is the same as the one extracted from the high-temperature Hall effect data (main panel of Fig.(1) while the paramagnetic ground state has a "large" Fermi surface which presumably counts the new heavy-fermion states injected by the local moments. 
The crossover line $T_{\text {Hall }}(B)$ (Fig. [3) is then interpreted as the finite temperature signature of the "jump" in the Fermi surface volume. It delineates the position at which a new large Fermi surface emerges in the incoherent electron fluid. It is interesting that this pre-cursor to heavy quasiparticle formation takes place at temperatures well above the temperature $T^{*}$ (dashed black curve in Fig. 3a) below which the coherent LFL develops. The existence of a large Fermi surface in the absence of well-defined quasiparticles is well known in one-dimensional Luttinger liquids ${ }^{23}$. It is also reminiscent of the marginal Fermi liquid behaviour in cuprate superconductors, where a large Fermi surface is seen in the angle-resolved photoemission studies, but the scattering rate grows linearly, rather than quadratically, in temperature 24.25 . Note also that the crossover line $T_{\text {Hall }}(B)$ does not follow the antiferromagnetic transition line $T_{N}(B)$ (Fig.33). Indeed, within experimental resolution, the initial Hall coefficient shows no change at the zero field Néel temperature of $70 \mathrm{mK}$ (Fig.17). This behaviour contrasts dramatically with that expected in an itinerant SDW, where changes in the Hall coefficient should coincide with the Néel transition - as is indeed observed for $\mathrm{Cr}_{1-x} \mathrm{~V}_{x}$ (refs. 22 and 26). Thus we may discard the possibility that the observed crossover in the Hall coefficient of $\mathrm{YbRh}_{2} \mathrm{Si}_{2}$ is due to a unit-cell doubling in a symmetry breaking antiferromagnetic transition. Even though the crossover at $T_{\text {Hall }}(B)$ broadens rapidly with temperature [cf. FWHM $(T)$ in the inset of Fig. [3a and width of $d \gamma(B) / d B$ in Fig. [3] $\mathrm{b}$, so that it can not be followed beyond about $0.5 \mathrm{~K}$, the additional contribution $\Delta R_{H}$ to the initial Hall coefficient (main panel of Fig.17) which we attribute to fluctuations of the Fermi surface volume can be discerned up to much higher temperatures of order $10 \mathrm{~K}$. This is precisely the temperature below which NFL behaviour is observed in thermodynamic and dynamical properties9.12. This observation makes it very tempting to hold fluctuations of the Fermi surface volume responsible for the NFL behaviour observed over this same temperature window. The fact that the NFL behaviour is observed in the entire phase diagram above $T_{N}$ and $T^{*}$ (and below $10 \mathrm{~K}$ ) can be related to the broadness of the crossover. Interestingly, also the spin fluctuation scale $T_{0}$ extracted from a logarithmic fit $\Delta C_{p} / T \propto \ln \left(T_{0} / T\right)$ to the specific heat data for $0.3 \mathrm{~K}<T<10 \mathrm{~K}$ and the single-ion Kondo temperature (which marks the onset of magnetic screening) extracted from a magnetic entropy measurement are of the same order of magnitude 9.12 .

To summarize we observe a rapid crossover of the Hall coefficient as function of a control parameter. By extrapolation to $T=0$ of both the Hall crossover and the magnetic phase 
transition $\underline{12}$, we infer that a large jump of the Hall coefficient occurs at the QCP. We expect this new insight, made possible primarily by the absence of superconductivity, to have broad implications for other strongly correlated electron systems ${ }^{27}$.

\section{Methods}

\section{Anomalous Hall effect}

In general, the Hall effect of materials containing localized magnetic moments is dominated at high temperatures by an anomalous Hall effect produced by the left-right asymmetry in incoherent electron scattering processes $2 \frac{28}{2}$. The initial or linear-response Hall coefficient $R_{H}$ (Hall coefficient in zero-field limit) scales for many materials with the product of electrical resistivity $\rho$ and magnetic susceptibility $\chi$,

$$
R_{H}=R_{0}+C \rho \chi
$$

where $R_{0}$ is the normal Hall coefficient and $C$ is a constant ${ }^{28}$. The term $C \rho \chi$ represents the anomalous Hall effect due to intrinsic scattering. The temperature-independent extrinsic anomalous Hall coefficient $R_{e x}$ due to skew scattering by residual defects may be estimated from

$$
R_{e x}=C \rho_{0} \chi_{0}
$$

where $\rho_{0}$ is the residual resistivity and $\chi_{0}$ the residual volume magnetic susceptibility $\underline{28}$. A model including crystalline electric field effects valid in the incoherent regime ${ }^{29}$, on the other hand, predicts

$$
R_{H}=R_{0}+R_{s} \chi
$$

instead of Eq. (3). Here $R_{s}$ is a constant and $R_{s} \chi$ the anomalous Hall-effect term.

In Fig. 1h we have shown that also in $\mathrm{YbRh}_{2} \mathrm{Si}_{2}$ the high-temperature Hall coefficient is dominated by the anomalous Hall effect. Between 7 and $300 \mathrm{~K}$ (90 and $300 \mathrm{~K}$ ), Eq. (3) [Eq. (5)] holds (cf. inset of Fig. (1) $)$. The $R_{0}$ value obtained for both models is $(2.4 \pm 0.1) \times$ $10^{-10} \mathrm{~m}^{3} / \mathrm{C}$ which corresponds, in a simple one band model, to a charge carrier concentration of $2.6 \times 10^{28} \mathrm{~m}^{-3}$ (approximately 2 holes per formula unit of $\mathrm{YbRh}_{2} \mathrm{Si}_{2}$ ). Considering only the magnetic contribution to $\rho$ in Eq. (3) yields similar values for $R_{0}$ (ref. 18). Below about $1 \mathrm{~K}$, where the extrapolation of the fit according to Eq. (3) (red dashed curve in Fig.(16) becomes temperature independent and saturates at the value of the normal Hall coefficient $R_{0}$, the intrinsic anomalous Hall effect is negligible. The extrinsic anomalous Hall effect 
estimated from Eq. (41) with $\rho_{0} \approx 1 \mu \Omega \mathrm{cm}$ and $\chi_{0}=0.0035(B \| c, T=40 \mathrm{mK})$ (ref. 12) is less than $4 \%$ of $R_{0}$ and thus plays a negligible role. Therefore, below about $1 \mathrm{~K}$, the initial Hall coefficient of $\mathrm{YbRh}_{2} \mathrm{Si}_{2}$ is essentially free of any anomalous contribution.

The anomalous Hall effect in finite magnetic fields may, in analogy with Eq. (3), be estimated from

$$
\rho_{H, a}(B)=C \rho(B) \mu_{0} M(B)
$$

where $\rho(B)$ and $M(B)$ are the field-dependent electrical resistivity and magnetization, respectively.

For $\mathrm{YbRh}_{2} \mathrm{Si}_{2}, \rho(B)$ (not shown) and $M(B)$ (ref. 12) have been measured in the relevant geometry $(B \| c$, current $I \perp c)$. For the parameter $C$ we use the value extracted from the temperature dependence of the initial Hall coefficient (inset of Fig. 1a). $\rho_{H, a}$ is less than 20\% of $\rho_{H}$ at all temperatures and fields.

Correspondence and requests for materials should be addressed to S. P. (paschen@cpfs.mpg.de).

Acknowledgements We acknowledge discussions with P. Bühler, J. Custers, C. Langhammer, C. Pépin, A. Rosch, A. Schofield, M. Vojta, A. Tsvelik, and F. Weickert. Part of the work at Dresden was supported by the Fonds der Chemischen Industrie. P. C. and Q. S. are supported by the National Science Foundation. The work at Rice University was partially supported by the Welch Foundation, and TCSAM. 
1. Martin, R. M. Fermi-surface sum rule and its consequences for periodic Kondo and mixedvalence systems. Phys. Rev. Lett. 48, 362-365 (1982).

2. Fulde, P. in Narrow-Band Phenomena - Influence of Electrons with both Band and Localized Character (ed. Fuggle, J. C.) 27-29 (Plenum Press, New York, 1988).

3. Oshikawa, M. Topological approach to Luttinger's theorem and Fermi surface of a Kondo lattice. Phys. Rev. Lett. 84, 3370-3373 (2000).

4. Hertz, J. A. Quantum critical phenomena. Phys. Rev. B 14, 1165-1184 (1976).

5. Millis, A. J. Effect of a nonzero temperature on quantum critical points in itinerant fermion systems. Phys. Rev. B 48, 7183-7196 (1993).

6. Schröder, A. et al. Onset of antiferromagnetism in heavy-fermion metals. Nature 407, 351-355 (2000).

7. Coleman, P., Pépin, C., Si, Q. \& Ramazashvili, R. How do Fermi liquids get heavy and die? J. Phys.: Condens. Matter 13, R723-R738 (2001).

8. Si, Q., Rabello, S., Ingersent, K. \& Smith, J. L. Locally critical quantum phase transitions in strongly correlated metals. Nature 413, 804-808 (2001).

9. Trovarelli, O. et al. $\mathrm{YbRh}_{2} \mathrm{Si}_{2}$ : Pronounced non-Fermi-liquid effects above a low-lying magnetic phase transition. Phys. Rev. Lett. 85, 626-629 (2000).

10. Ishida, K. et al. $\mathrm{YbRh}_{2} \mathrm{Si}_{2}$ : Spin fluctuations in the vicinity of a quantum critical point at low magnetic fields. Phys. Rev. Lett. 89, 107202 (2002).

11. Ishida, K. et al. Low-temperature magnetic order and spin dynamics in $\mathrm{YbRh}_{2} \mathrm{Si}_{2}$. Phys. Rev. $B$ 68, 184401 (2003).

12. Gegenwart, P. et al. Magnetic-field induced quantum critical point in $\mathrm{YbRh}_{2} \mathrm{Si}_{2}$. Phys. Rev. Lett. 89, 056402 (2002).

13. Küchler, R. et al. Low-temperature thermal expansion and magnetostriction of $\mathrm{YbRh}_{2}\left(\mathrm{Si}_{1-x} \mathrm{Ge}_{x}\right)_{2}$ (x=0 and 0.05). J. Magn. Magn. Mater. 272-276, 229-230 (2004).

14. Custers, J. et al. The break-up of heavy electrons at a quantum critical point. Nature 424, 524-527 (2003).

15. Stewart, G. R. Non-Fermi-liquid behavior in $d$ - and f-electron metals. Rev. Mod. Phys. 73, $797-855$ (2001). 
16. Senthil, T., Vojta, M. \& Sachdev, S. Weak magnetism and non-Fermi liquids near heavyfermion critical points. Phys. Rev. B 69, 035111 (2004).

17. Paschen, S. et al. Hall effect of the NFL compound $\mathrm{YbRh}_{2} \mathrm{Si}_{2}$. Acta Physica Polonica B 34, 359-362 (2003).

18. Paschen, S. et al. Anomalous Hall effect in $\mathrm{YbRh}_{2} \mathrm{Si}_{2}$. To appear in Physica B (Proc. SCES04).

19. Anderson, P. W. Hall effect in the two-dimensional Luttinger liquid. Phys. Rev. Lett. 67, 2092-2094 (1991).

20. Nakajima, Y. et al. Normal-state Hall angle and magnetoresistance in quasi-2D heavy fermion $\mathrm{CeCoIn}_{5}$ near a quantum critical point. J. Phys. Soc. Jpn. 73, 5-8 (2004).

21. Custers, J. Quantum-Critical Behavior in the Heavy-Fermion Compounds $\mathrm{YbRh}_{2} \mathrm{Si}_{2}$ and $\operatorname{CeIn}_{3-x} S n_{x}$. PhD thesis, TU Dresden, Germany, (2004).

22. Lee, M., Husmann, A., Rosenbaum, T. F. \& Aeppli, G. High resolution study of magnetic ordering at absolute zero. Phys. Rev. Lett. 92, 187201 (2004).

23. Haldane, F. D. M. 'Luttinger liquid theory' of one-dimensional quantum fluids: I. Properties of the Luttinger model and their extension to the general 1D interacting spinless Fermi gas. $J$. Phys. C: Solid State Phys. 14, 2585-2609 (1981).

24. Varma, C. M., Littlewood, P. B., Schmitt-Rink, S., Abrahams, E. \& Ruckenstein, A. E. Phenomenology of the normal state of $\mathrm{Cu}-\mathrm{O}$ high-temperature superconductors. Phys. Rev. Lett. 63, 1996-1999 (1989).

25. Valla, T. et al. Evidence for quantum critical behaviour in the optimally doped cuprate $\mathrm{Bi}_{2} \mathrm{Sr}_{2} \mathrm{CaCu}_{2} \mathrm{O}_{8+\delta}$. Science 285, 2110-2113 (1999).

26. Yeh, A. et al. Quantum phase transition in a common metal. Nature 419, 459-462 (2002).

27. Balakirev, F. F. et al. Signature of optimal doping in Hall-effect measurements on a hightemperature superconductor. Nature 424, 912-915 (2003).

28. Fert, A. \& Levy, P. M. Theory of the Hall effect in heavy-fermion compounds. Phys. Rev. B 36, 1907-1916 (1987).

29. Kontani, H., Miyazawa, M. \& Yamada, K. Theory of anomalous Hall effect in a heavy fermion system with a strong anisotropic crystal field. J. Phys. Soc. Jpn. 66, 2252-2255 (1997).

30. Norman, M. R., Si, Q., Bazaliy, Y. B. \& Ramazashvili, R. Hall effect in nested antiferromagnets near the quantum critical point. Phys. Rev. Lett. 90, 116601 (2003). 


\section{Supplementary Methods 1}

\section{Hall response from $B_{2}$}

In the crossed-field experiment, we establish a steady state electrical current along $\hat{x}$, and apply two perpendicular magnetic fields: a tuning field $B_{2}$ along $\hat{x}$ and a probing field $\delta B_{1}$ along $\hat{z}$. We measure the transverse (Hall) voltage along $\hat{y}$. In our experiment, $\hat{x}, \hat{y}, \hat{z}$ are chosen to be the principal axes and, moreover, the crystal is isotropic in the $x y$-plane. The currents and voltages are related by the conductivity tensor:

$$
\left(\begin{array}{c}
J_{x} \\
J_{y} \\
J_{z}
\end{array}\right)=\left(\begin{array}{ccc}
\sigma_{\|} & \sigma_{x y} & 0 \\
& & \\
-\sigma_{x y} & \sigma_{\|} & \sigma_{y z} \\
0 & -\sigma_{y z} & \sigma_{\perp}
\end{array}\right) \times\left(\begin{array}{c}
E_{x} \\
E_{y} \\
\\
E_{z}
\end{array}\right) .
$$

Here we have used $\sigma_{x z}=0$, reflecting the absence of magnetic field along $\hat{y}$, and adopted $\sigma_{\|}$to denote $\sigma_{x x}$ and $\sigma_{y y}$ (the slight difference between $\sigma_{x x}$ and $\sigma_{y y}$ induced by a finite $B_{2}$ can be easily incorporated in our discussion and will not affect our conclusion below) and $\sigma_{\perp}=\sigma_{z z}$. Solving these equations under the conditions $J_{z}=J_{y}=0$, we find

$$
R_{H} \equiv \lim _{B_{1} \rightarrow 0} \frac{E_{y}}{J_{x} B_{1}}=\lim _{B_{1} \rightarrow 0} \frac{\sigma_{x y}}{\sigma_{\|}^{2} B_{1}} \times\left[1+\frac{\sigma_{y z}^{2}}{\sigma_{\perp} \sigma_{\|}}\right]^{-1} .
$$

On the right hand side of Eq. (8) , the first factor describes the Hall constant in a gedanken setup in which the field $B_{2}$ does not produce any Lorentz force while fulfilling the role of tuning the underlying state. The second factor in principle differs from 1. For an order-ofmagnitude estimate, we can ignore the $x y$ vs $z$ anisotropy and take $\frac{\sigma_{y z}^{2}}{\sigma_{\perp} \sigma_{\|}} \approx\left(\frac{\rho_{H}}{\rho}\right)^{2}$. Given that the field $B_{2}$ we have applied in the crossed-field experiment is much smaller than the largest field $B_{1}$ used in our single-field experiment, $\rho_{H}$ here should be much smaller than $6.5 \times 10^{-10} \Omega \mathrm{m}$, the value shown in Fig. 2a of our Letter to Nature for the temperature range $T<200 \mathrm{mK}$. The longitudinal resistivity $\rho$ is of the order $10^{-8} \Omega \mathrm{m}$. The second factor on the right hand side of Eq. (8) , then, differs from 1 by much less than $5 \times 10^{-3}$.

To summarize, to an accuracy much better than $0.5 \%$, the initial slope of the $\rho_{H}$ vs $B_{1}$ plot in the crossed-field experiment measures the linear-response Hall coefficient of the state reached by the finite tuning field $B_{2}$. 


\section{Supplementary Methods 2}

\section{Kubo formalism for differential Hall coefficient}

The concept of a differential Hall coefficient is intimately related to underlying current correlations inside the material. In thermal equilibrium, the "fluctuation dissipation theorem" gives rise to a unique link between the differential response of a system to a field and the fluctuations of the variable that is coupled to the field. These relationships are determined by the so-called Kubo formalism ${ }^{1}$ For example, the differential magnetic susceptibility of a material is directly proportional to the two-point spin-spin correlation function. The orbital part of the differential Hall conductivity of a metal can be regarded as a current susceptibility to small changes in the magnetic field ${ }^{2}$,

$$
d^{2} J_{x}=\delta B \delta E_{y}\left(\frac{d \sigma_{x y}}{d B}\right)_{\text {orb }}
$$

where $\delta E_{y}$ is the electric field along the $y$-axis and $J_{x}$ is the Hall current density along the $x$-axis. In simple metals, the Lorentz force acting on the electrons grows linearly with the

applied field, giving rise to a simple linear response $\sigma_{x y}=\frac{d \sigma_{x y}}{d B} B$. Near a QCP, the velocities of the quasiparticles are highly sensitive to the field-tuned ground state, so the Lorentz force changes with the tuning field obliging us to take the field dependence of the differential Hall coefficient into account.

Fortunately, under general conditions, the Kubo formula enables us to relate the differential Hall response to the three-point current correlation function ${ }^{2}$

$$
Q_{\alpha \beta \gamma}(q, B)=\left\langle B\left|J_{\alpha}(q) J_{\beta}(-\vec{q}) J_{\gamma}(\omega)\right| B\right\rangle
$$

where $q \equiv(\vec{q}, \omega)$ describes the respective frequency $\omega$ and wavevector $\vec{q}$ of a probe electric and magnetic field, $J_{\alpha}$ is the current density in the $\alpha$ direction at the appropriate frequency and wavevector and $|B\rangle$ denotes the field-tuned ground state of the system. All "anomalous" contributions to the ground-state Hall conductance can be included into the above expression, if the $J_{\alpha}$ are taken to be the full current operators, taking into account the momentum dependence of the hybridization between conduction and $f$ electrons..$^{3}$ The relation

$$
Q_{\alpha \beta \gamma}(q, B)-Q_{\alpha \beta \gamma}(0, B)=\omega\left(q_{\alpha} \delta_{\beta \gamma}-q_{\beta} \delta_{\alpha \gamma}\right)\left(\frac{d \sigma_{x y}}{d B}\right)_{\text {orb }}
$$

determines the orbital part of the differential Hall conductivity. In practice, it is more convenient to measure the differential Hall resistivity, which is simply related to the the 
differential Hall conductivity via the relation $\frac{d \rho_{y x}}{d B} \equiv \tilde{R}_{H}(B)=\rho^{2} \frac{d \sigma_{x y}}{d B}$, where $\rho$ is the electrical resistivity and $\rho_{y x}\left(=\rho_{H}\right)$ the Hall resistivity. In our experiments the geometry between the probe field $\delta B_{1}$, Hall voltage and injected current is the same in both the transverse and longitudinal field-tuning configurations, so both $\left[\frac{\partial \sigma_{H}\left(B_{1}\right)}{\partial B_{1}}\right]_{\text {orb }}=\left[\tilde{R}_{H}\left(B_{1}\right)\right]_{\text {orb }} / \rho^{2}$ in the transverse and $\sigma_{x y}\left(B_{2}, B_{1}\right) /\left.B_{1}\right|_{B_{1} \rightarrow 0}=R_{H}\left(B_{2}\right) / \rho^{2}$ in the longitudinal configuration measure the evolution of the same current correlator through the QCP.

1. Kubo, R. Statistical-mechanical theory of irreversible processes. 1. General theory and simple applications to magnetic and conduction problems. J. Phys. Soc. Jpn. 12, 570-586 (1957).

2. Voruganti, P., Golubentsev, A. \& John, S. Conductivity and Hall effect in the two-dimensional Hubbard model. Phys. Rev. B 45, 13945-13961 (1992).

3. Kontani, H. \& Yamada, K. Theory of anomalous Hall effect in heavy fermion system. J. Phys. Soc. Jpn. 63, 2627 (1994). 
FIG. 1: Temperature dependence of the Hall effect of $\mathrm{YbRh}_{2} \mathrm{Si}_{2}$. a, Temperature-dependent initial Hall coefficient $R_{H}(T)$, obtained from the initial slope of Hall resistivity vs field isotherms (Fig. 27). The red curve corresponds to the red fit to the data from the inset. $\Delta R_{H}$ designates the difference between the data and the fit. The green triangles correspond to $R_{H}$ data obtained from the crossed-field experiment for large values of the tuning field $B_{2}\left[R_{H}^{\infty}\right.$ values of fits to $R_{H}\left(B_{2}\right)$, cf. text and Fig.20], suggesting that the Fermi surface volume is distinctly larger in the fieldinduced paramagnetic than in the antiferromagnetic state. Inset in $\mathbf{a}$, Initial Hall coefficient $R_{H}$ vs product of electrical resistivity $\rho$ and magnetic susceptibility $\chi$ (lower axis) and vs $\chi$ (upper axis), where temperature is an internal parameter. The full red (black) line is a linear fit according to the anomalous Hall-effect relation Eq. (3) [Eq. (5)] to the data between 7 and $300 \mathrm{~K}$ (90 and $300 \mathrm{~K}$ ), the dashed lines are the extrapolations to $T=0$. b. Cotangent of the Hall angle $\cot \Theta_{H}$ $\left(\equiv \frac{\rho}{R_{H} B}\right)$ as a function of $T^{2}$, taken at $B=1 \mathrm{~T}$. The red line (also in the inset) corresponds to a fit, $\cot \Theta_{H}=C_{1}+C_{2} T^{2}$, where $C_{1}$ and $C_{2}$ are constants. Inset in $\mathbf{b}$, Difference between data and fit (red line) of main panel. The black line is a guide to the eye. Below $0.7 \mathrm{~K}$, the data deviate considerably from the fit. The green squares correspond to $\cot \Theta_{H}$ data obtained from the crossed-field experiment at the respective crossover fields $\left(B_{2}=B_{0}\right)$, indicating that, closer to the QCP, these deviations are even stronger. Thus, the $\cot \Theta_{H}=C_{1}+C_{2} T^{2}$ behaviour appears to be a property of the regime at elevated temperatures where quantum critical fluctuations start to influence the physical properties, but it does not extend over the entire temperature region down to the QCP. 
FIG. 2: Magnetic field dependence of the Hall effect of $\mathrm{YbRh}_{2} \mathrm{Si}_{2}$. a, Single-field experiment. Typical isotherms of the Hall resistivity $\rho_{H}$, corrected for its anomalous contribition $\rho_{H, a}(B)$ [Eq. (6)] , vs magnetic field $B_{1}=\mu_{0} H_{1}$ ( $\| c$-axis). The solid curves represent best fits, $\int \tilde{R}_{H}(B) d B$ (see text), to the data. The derivative of the fit at $75 \mathrm{mK}$ is plotted on the right axis. b, Crossed-field experiment. Initial slope $R_{H}$, normalized to its value at the crossover field $B_{0}$, of all measured $\rho_{H}$ vs $B_{1}$ curves as a function of $B_{2} / B_{0}$, at $45,65,75$, and $93 \mathrm{mK}$. The solid lines represent best fits (see text) to the data. $R_{H}$ decreases by a factor of $\approx 1.5$ upon going from the zero-field antiferromagnetic to the field-induced paramagnetic state. In an SDW picture $R_{H}$ is expected to evolve as the magnetic order parameter ${ }^{7}$. In $\mathrm{YbRh}_{2} \mathrm{Si}_{2}$ the ordered moment at $B=0$ was estimated to be $\approx 0.002 \mu_{B} / \mathrm{Yb}$ (ref. 11). Thus, the change in $R_{H}$ corresponds to a factor of $\approx 750 / \mu_{B}$. The corresponding change of $R_{H}$ by a factor of $\approx 30 / \mu_{B}$ observed for the SDW system $\mathrm{Cr}_{1-x} \mathrm{~V}_{x}$ (refs. 22 and 26) was already considered a giant effect, possibly connected with bandstructure nesting effects ${ }^{30}$. By comparison, the effect in $\mathrm{YbRh}_{2} \mathrm{Si}_{2}$ is about 25 times as large. Even in the absence of both experimental and theoretical studies of the electronic bandstructure of $\mathrm{YbRh}_{2} \mathrm{Si}_{2}$ we judge this effect far too large to be accounted for within an SDW picture. The same agrument holds even if the second order transition observed in the measured temperature range $(>15 \mathrm{mK})$ turned over into a first order one at $T<15 \mathrm{mK}$. The inset in $\mathbf{b}$ displays $\rho_{H}$ vs $B_{1}$ curves at three different values of the tuning field $B_{2}=\mu_{0} H_{2}(\perp c$-axis $)$ at $65 \mathrm{mK}$. The solid lines represent best fits, as in a. Similar data have been obtained at the other temperatures (not shown). The sketches in a and b illustrate the experimental set-up. 
FIG. 3: Temperature-field phase diagrams of $\mathrm{YbRh}_{2} \mathrm{Si}_{2}$. a, The red data points correspond to the $B_{0}$ values (crossover positions in the Hall-effect measurements) determined from the fits to the data in Fig. 2a (single-field experiment). Note that the horizontal bars represent the error in the determination of $B_{0}$ rather than the width of the crossover. The red dotted line denoted $T_{\text {Hall }}$ is the best linear fit to all data up to $0.5 \mathrm{~K}$. It extrapolates at zero temperature to $\approx 0.7 \mathrm{~T}$, the critical field $B_{1 c}$ for the direction parallel to the $c$-axis. The green data points correspond to $11 B_{0}$ determined from the fits to the data in Fig.20 (crossed-field experiment). The full and dotted black curve represent the field dependence of the Néel temperature $T_{N}$ and the crossover temperature $T^{*}$ to a $\Delta \rho \propto T^{2}$ law, respectively, as determined from iso-field $\rho(T)$ data 12 . The latter differs qualitatively from the cross-over line determined from a scaling analysis of both specific heat and resistivity data, yielding $T_{\text {cross }} \propto\left(B-B_{c}\right)$ (ref. 14). The inset shows the full width at half maximum (FWHM) of $d \tilde{R}_{H}\left(B_{1}\right) / d B_{1}$ in a log-log plot (red points). The red solid line, $\propto T^{a}, a=0.5 \pm 0.1$, is a best fit to these data. As in the main panel, the green dots correspond to the crossed-field experiment. For both the main panel and the inset, the red and green data points agree within the error bars. $\mathbf{b}, 3 \mathrm{D}$ representation of the field derivative of the crossover function $\gamma(B)$ defined in the text. The coloured curves represent arbitrary isotherms of $d \gamma(B) / d B$, obtained using both the $B_{0}(T)$ fit of a and a power law fit to the corresponding $p(T)$ data (not shown). The field $B$ corresponds to $B_{1} \| c$ or to $11 B_{2} \perp c$. The positions $B_{0}$ are designated by broken drop lines and the black dotted line denoted $T_{\text {Hall }}$ in the $T-B$ plane. The antiferromagnetic phase and the region where $\Delta \rho \propto T^{2}$ are marked as black and hatched areas, respectively, in the $T-B$ plane. At the lowest temperatures, $d \gamma(B) / d B$ may be interpreted as indicating the change of the effective carrier concentration. In the limit $T \rightarrow 0, d \gamma(B) / d B$ is a $\delta$-function (dotted line in the $T=0$ plane), separating the states of small and large Fermi surface (FS) at $B=B_{1 c}=11 B_{2 c}$. 

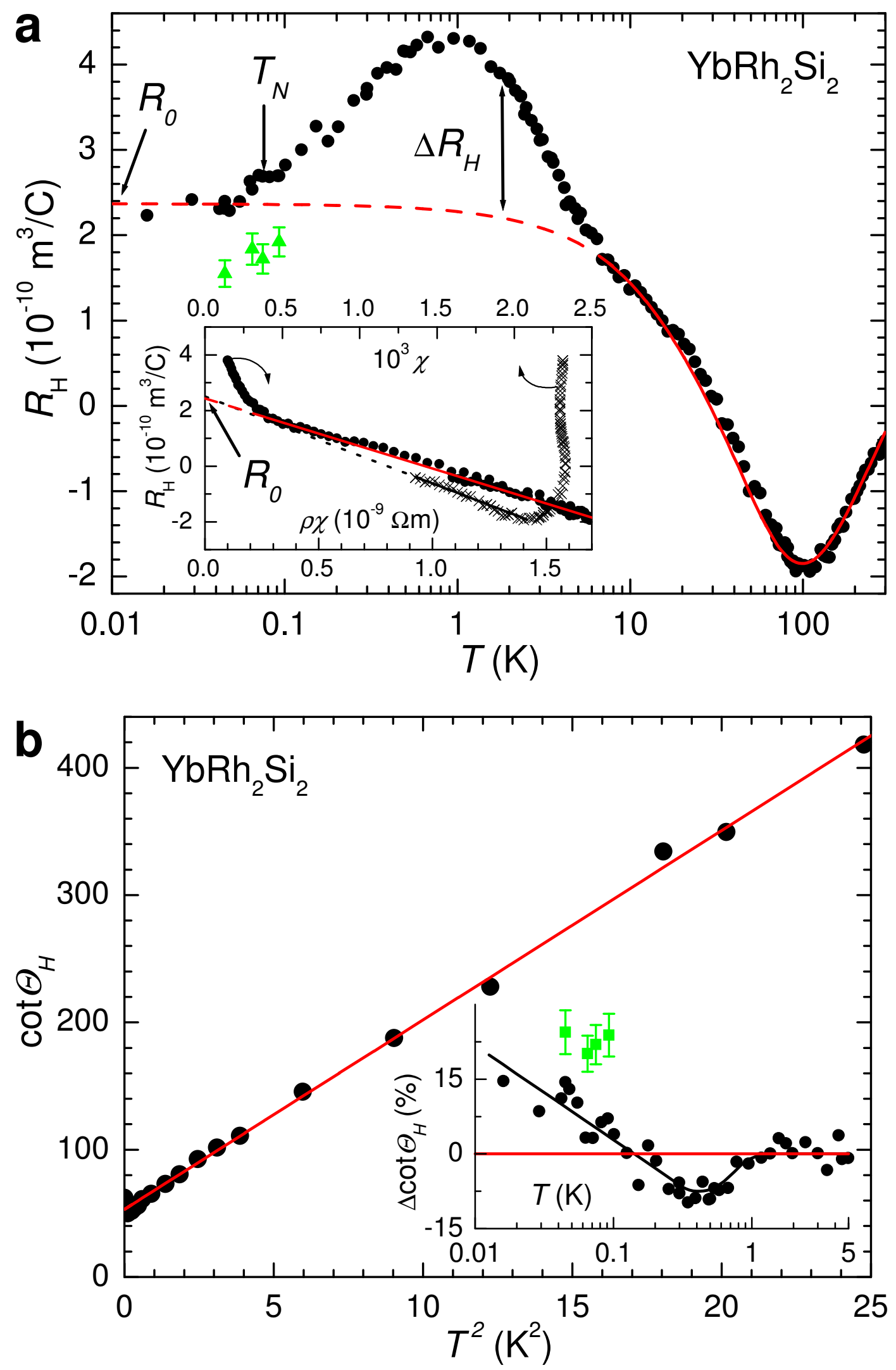

Figure 1 

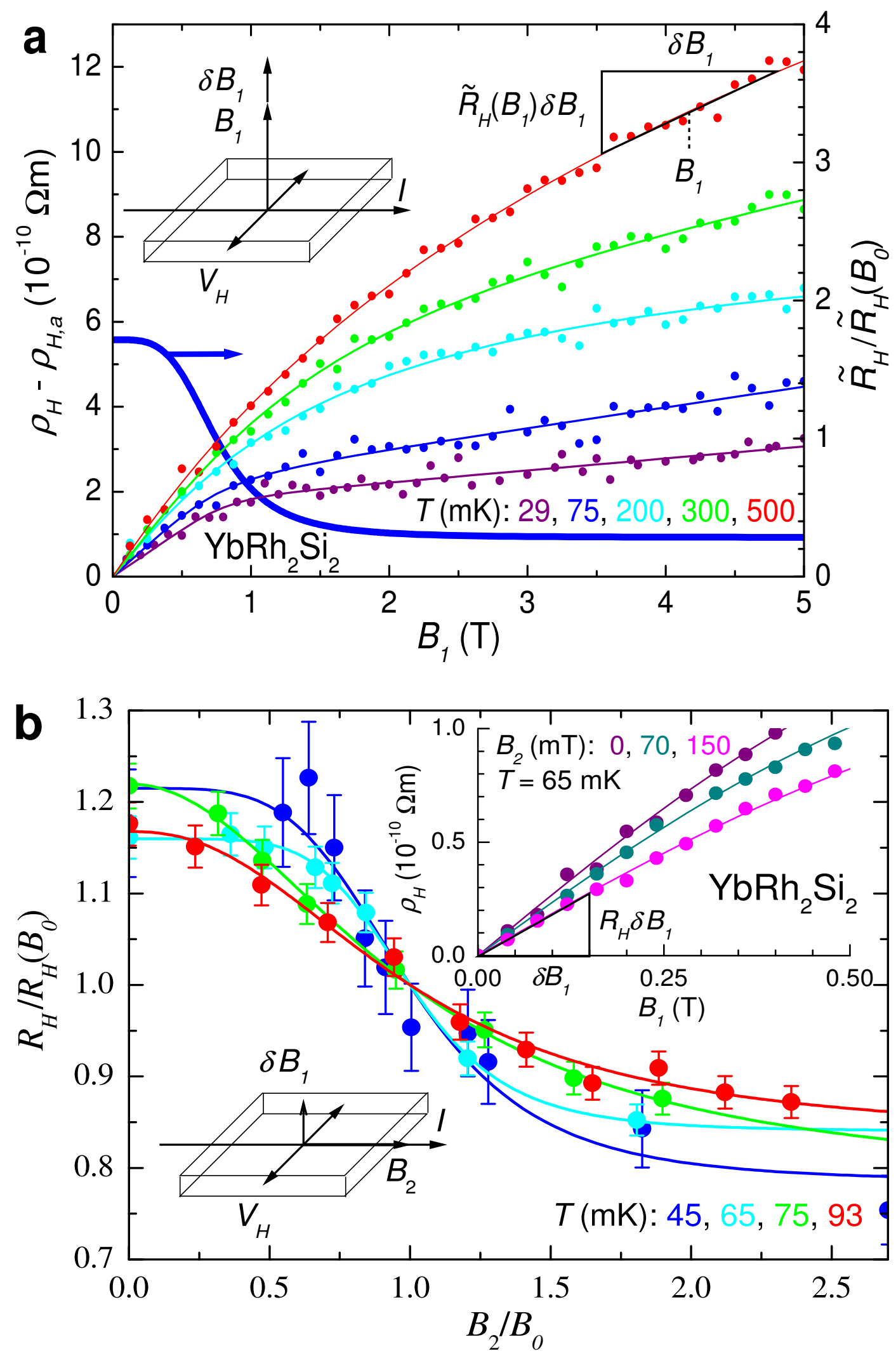

Figure 2 

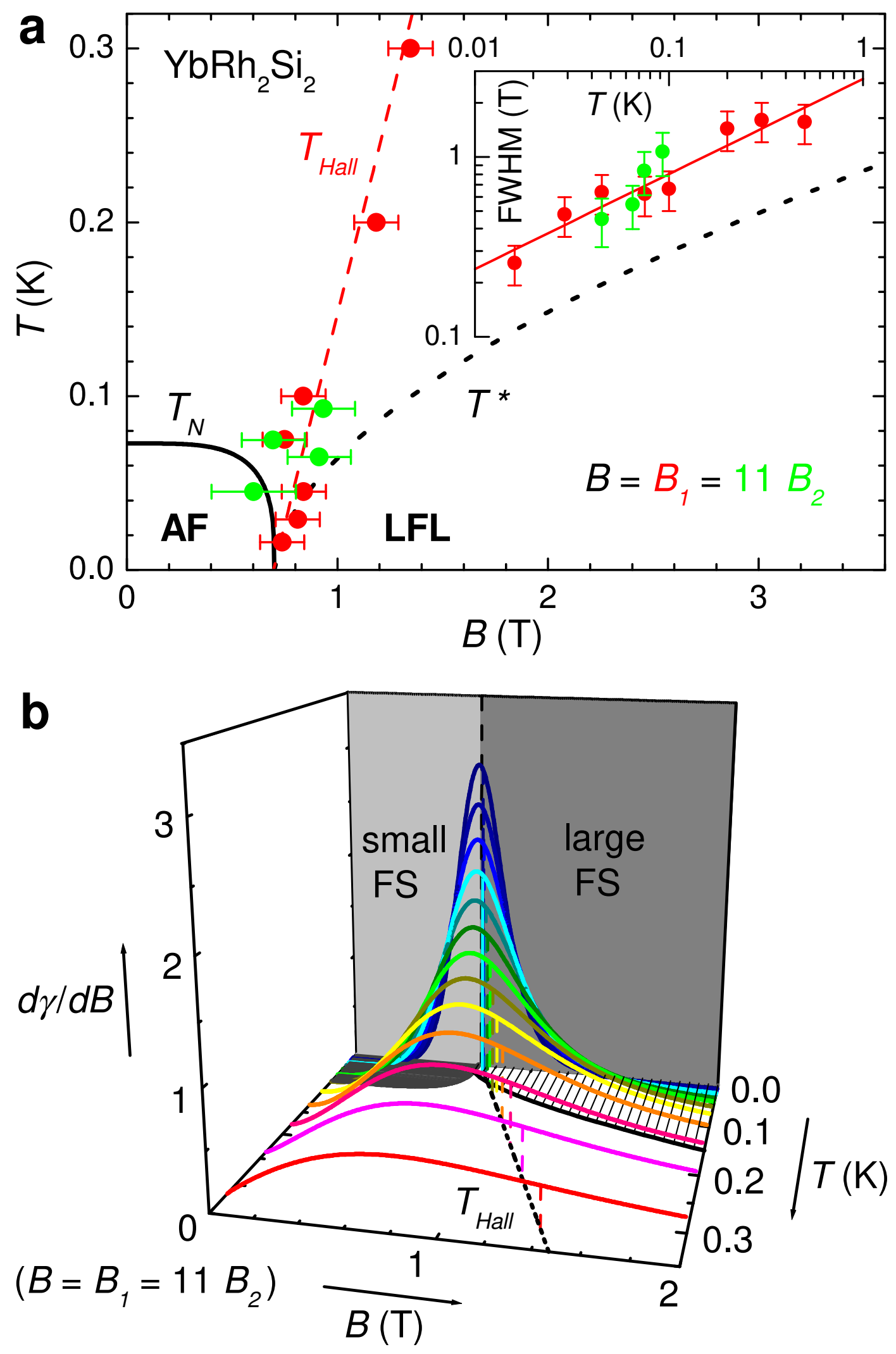

Figure 3 